These fungi form symbioses - mycorrhizas - with plant roots which help plants to acquire mineral nutrients from the soil and also determine plant biodiversity and ecosystem function ${ }^{8}$. They are truly ancient, having remained largely morphologically unchanged since plants first colonized the land around 400 million years ago ${ }^{9}$. No sexual stage in the Glomales life cycle has been observed.

Polymorphism in the ribosomal DNA encoding the internal transcribed spacer (ITS), and the $5.8 \mathrm{~S}$ and $18 \mathrm{~S}$ subunits, occurs inside individual spores of fungi of the genus Glomus $^{10,11}$. This is unusual because it is widely thought that sequences of multiple copies of ribosomal DNA are kept the same by a process known as concerted evolution ${ }^{12}$, in which the joint evolution of two or more related genes occurs as if they constitute a single locus. It is on the assumption of concerted evolution that the ribosomal sequences are so widely used for taxonomy and phylogenetics $^{13}$. The existence of different sequences of ribosomal genes in Glomus implies that recombination does not occur, because the mechanisms that keep the sequences of rDNA copies the same operate most frequently during recombination events.

Both of the new studies ${ }^{6,7}$ looked at another fungus within the Glomales, Scutellospora castanea, and worked with exactly the same isolate ${ }^{14}$ which was maintained in culture in the roots of plants. Using the polymerase chain reaction (PCR), six different ITS sequences and 13 of the $18 \mathrm{~S}$ gene were picked out, respectively, from genomic DNA and from clones in an $S$. castanea genomic DNA library. This does not mean that variation is limited to six different sequences of each. Hijri et al. ${ }^{6}$ confirmed that several different ITS sequences (including those of the $5.8 \mathrm{~S}$ gene) occur in a single spore, but that each spore of this isolate does not have the same complement of the different sequences (Fig. 1a).

The Glomales are coenocytes - that is, many nuclei are enclosed within one cell wall - and it could be that different rDNA sequences occur on different nuclei in which the rDNA sequences have diverged due to a lack of recombination. To test this possibility, Hijri et al. ${ }^{6}$ diluted nuclear suspensions from single spores to an expected one-nucleusper-sample and performed PCR with specific primers that amplify the different rDNA sequences. Their results indicate that, indeed, different rDNA sequences are carried on different nuclei (Fig. 1a); so it seems that an individual of these fungi is, in genetic terms, actually a population of discrete nuclei.

Perhaps more astounding, however, is just how divergent the ribosomal gene sequences are. Hosny et al. ${ }^{7}$ carried out a phylogenetic analysis of $18 \mathrm{~S}$ sequences from S. castanea and from species of each of the other Glomales genera. Most sequences grouped in the genus Scutellospora, but two others from the same $S$. castanea isolate were so divergent that they clustered in the genus Glomus(Fig. 1b).

A contaminant in the genomic library, perhaps? It seems not, because the Glomuslike rDNA sequences were successfully amplified again from single spores of this isolate of $S$. castanea. According to previous phylogenetic analyses of the Glomales, the family known as Glomaceae, which contains the genus Glomus, diverged from the other mycorrhizal fungi that were later to form the Acaulosporaceae and the Gigasporaceae (containing the genus Scutellospora), between 353 and 367 million years ago ${ }^{15}$.

How then can we explain the observations reported in these two studies? The results support the idea that genetic drift the random change of allelic frequencies in a population - has occurred in the absence of recombination, leading to genetically divergent nuclei. But at the same time it seems

\title{
Polar endeavours
}

Almost exactly 85 years ago, Sir Ernest Shackleton set out to cross the Antarctic continent. After his ship was frozen into the ice pack, the expedition waited and worked through the long polar nights, then the long polar days, before Shackleton managed to get them all rescued, a story that continues to generate popular books ${ }^{1}$. In honour of his exploits, Shackleton had a lunar crater (just to the right of centre in this image of the lunar south pole) named after him. That crater is now getting attention as a part of another story that involves ice, long days and nights - and perhaps, ultimately, human exploration.

Lunar scientists have long known that because the Moon's axis of rotation is almost perpendicular to its path around the Sun, the polar regions could have high points that are permanently sunlit, and crater floors that are permanently in shadow. This false-colour image (inside the yellow line, and overlaid on a radar image of the region) shows the percentage of the lunar day for which a given location is illuminated (white, orange and red receive the most illumination, the clear regions receive none), based on a newly reported analysis of images taken in 1994 by the Clementine spacecraft ${ }^{2}$. A separate radar study ${ }^{3}$ has confirmed that many regions,

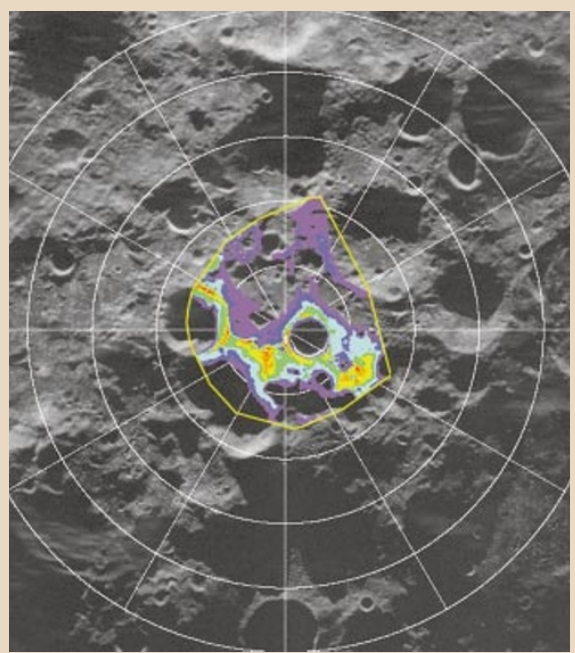

including the bottom of Shackleton Crater, are likely to be permanently shadowed. Conversely, some regions are sunlit most of the time and would be prime locations for lunar bases, or at least for solar arrays to support a polar base. For example, the white region at the left (poleward) edge of Shackleton Crater is sunlit more than $\mathbf{8 0 \%}$ of the time, and there is a ridge $10 \mathrm{~km}$ away that is sunlit $90 \%$ of the time that the first spot is not.

A polar base would be more attractive if the permanently shadowed crater floors have managed to cold-trap water ice, which could be mined for life support or fuel. Data from the neutron spectrometer aboard the Lunar Prospector spacecraft ${ }^{4}$ apparently showed abundant hydrogen at the poles, possibly, though not definitely, in the form of water ice; similarly, a Clementine radar experiment may ${ }^{5}$ or may not $^{6}$ have provided evidence for ice.

At the end of July, with its funding expired and its fuel nearly gone, Lunar Prospector will be crashed into a crater near the pole (the larger crater above and to the right of Shackleton) and Earthbound telescopes will search for evidence of water released by the impact ${ }^{7}$. The chances of the experiment detecting water are no better than $10 \%$, but this is just the next step in a search for the conditions that might make exploration of the lunar poles possible.

Timothy D.Swindle

Timothy D. Swindle is in the Lunar and Planetary Laboratory, University of Arizona, Tucson,

Arizona 85721-0092, USA.

e-mail: tswindle@U.Arizona.EDU

1.Alexander, C. The Endurance: Shackleton's Legendary Antarctic Expedition (Knopf, New York, 1998).

2. Bussey, D. B. J., Spudis, P. D. \& Robinson, M. Geophys. Res. Lett. 26, 1187-1190 (1999).

3. Margot, J. L.et al. Science 284, 1658-1660 (1999).

4. Feldman, W. C. et al. Science 281,1496-1500 (1998).

5. Nozette, S.et al. Science 274, 1495-1498 (1996).

6.Simpson, R. A. \& Tyler, G. L. J. Geophys. Res. 104, 3845-3862 (1999)

7.Goldstein, D. B. et al. Geophys. Res. Lett. 26, 1652-1656 (1999). 